\title{
PROPOSTA DE INSTALAÇÃO DE UM MÓDULO DE INJEÇÃO PROGRAMÁVEL EM UMA BANCADA DIDÁTICA DE COMBUSTÃO INTERNA DE 4 TEMPOS
}

PROPOSED INSTALLATION OF A PROGRAMMABLE INJECTION MODULE IN A 4-TIME

INTERNAL COMBUSTION DIDACTIC BANK

Mateus Pierdoná ${ }^{1}$

Márcio Kawamura²

\section{RESUMO}

O sistema de injeção eletrônica é utilizado em todas as montadoras de automóveis existentes. Entretanto, surgiu no mercado sistemas de injeção eletrônica programáveis, os quais podem fazer modificações em todo o sistema de injeção do motor, com o objetivo de trazer melhorias ao seu funcionamento em diferentes aplicações. O presente estudo está abordando um tema referente a instalação de um sistema de injeção programável em uma bancada de combustão interna de 4 tempos. O objetivo desta instalação é fazer com que esta bancada didática possa sofrer melhorias e alterações em seu funcionamento e trazer informações referentes ao motor. Visando esse objetivo, foi desenvolvido o projeto de implementação de um módulo de injeção eletrônica programável modelo Pandoo Proinject na bancada, possibilitando que os operadores possam realizar modificações, obter informações e fazer análises do funcionamento do motor. Foi realizado um projeto da instalação dos módulos necessários, orçamento dos componentes e uma imagem ilustrativa de como ficaria o sistema após a instalação. Através desta instalação é possível realizar diferentes programações em tempo real no sistema de injeção do motor, monitorar as informações dos sensores e atuadores, visualizar e alterar os mapas de funcionamento e trazer uma maior segurança.

Palavras-Chave: Pandoo Proinjec. Sensores. Atuadores. Motor. Instalação.

\footnotetext{
1 Graduado em Engenharia Mecânica pela Universidade Alto Vale do Rio do Peixe (UNIARP). Email: m_pierdona@hotmail.com.

2 Docente do curso de Engenharia Mecânica pela Universidade Alto Vale do Rio do Peixe (UNIARP). marciokw@hotmail.com.
} 


\section{ABSTRACT}

The electronic injection system is used in all existing car manufacturers. However, programmable electronic injection systems are available in the market, which can make modifications throughout the engine injection system, in order to bring improvements to its operation in different applications. The present study addresses a topic on the installation of a programmable injection system in a 4-stroke internal combustion engine. The purpose of this installation is to make this didactic stand can undergo improvements and changes in its operation and bring information about the engine. Aiming at this objective, the project was developed to implement a Pandoo Proinject programmable electronic injection module in the stand, allowing operators to make modifications, obtain information and perform analyzes of the engine operation. A project was carried out to install the required modules, the component budget, and an illustrative image of how the system would look after the installation. Through this installation it is possible to perform different schedules in the engine injection system, monitor the information of the sensors and actuators, view and change the operation maps and bring greater safety.

Keywords: Pandoo Proinject. Sensors. Actuators. Motor. Installation.

\section{INTRODUÇÃO}

A busca por motores visando uma maior economia, potência, torque, aproveitamento de combustível, desempenho e segurança, tem tido um crescimento considerável nos dias atuais. Existem algumas maneiras de se conseguir ao motor um melhor aproveitamento, porém na maioria das vezes isso é feito através de alterações na parte estrutural e mecânica do motor, como troca de peças, modificações no cabeçote, entre outros. Essas modificações podem ser prejudiciais ao motor, assim diminuindo sua vida útil, além de modificar sua originalidade.

O motor de combustão interna começou a ser concebido em 1862 quando o físico francês Alphonse Beau de Rochas propôs as condições necessárias para funcionamento de um motor quatro tempos, de combustão interna, funcionasse com um máximo de economia. Rochas descreveu também a sequência de eventos, por meio da qual a economia e a eficiência poderiam ser conseguidas. Essa sequência, que totalizava quatro tempos, é o que hoje ocorre basicamente em todo motor de combustão interna. Mas, Rochas não chegou a construir motor algum, apenas formulou as condições de funcionamento econômico que ele havia imaginado. O motor apenas construído experimentalmente em 1872, só foi 
realizado praticamente em 1876 por Nikolaus Otto, o qual determinou o ciclo teórico sob o qual trabalha o motor de combustão interna. Assim que a firma alemã Otto and Langen passou a fabricar os motores quatro tempos, de pistões moveis, ligados a uma árvore de manivelas, este motor passou a se chamar popularmente de motor Otto (ANDREOLI, 2009).

O motor ciclo Otto (motor de ignição por centelha) é um motor com pistão, com formação interna ou externa de mistura ar-combustível. A formação da mistura externa geralmente produz misturas homogêneas, enquanto a mistura interna é altamente heterogênea no momento da ignição. Tanto o tempo de formação da mistura quanto a distribuição do combustível na câmara de combustão são fatores importantes que influenciam o grau de homogeneização que pode ser atingido pela formação interna da mistura (BOSCH, 2005).

Os quatro tempos do motor, são: tempo de admissão, tempo de compressão, tempo de expansão e tempo de escape (BRUNETTI, 2012).

A visualização das informações dos sensores e atuadores de um motor de combustão interna é de extrema importância, pois através disso pode ser feito o monitoramento da rotação, temperatura, pressão de óleo e combustível, entre outros, facilitando na identificação de defeitos no motor caso haja alguma falha. Outro fator importante é a segurança do motor, pois se o mesmo trabalhar em temperaturas muito elevadas, não possuir lubrificação suficiente e trabalhar em rotações muito altas, poderá trazer danos ao seu funcionamento.

Nos dias de hoje os sistemas de injeção eletrônica equipam todos os veículos movidos por motores de ignição por faísca a quatro tempos (FAGGI, 2012). Os sistemas de injeção/ignição eletrônica programáveis funcionam da mesma forma que os sistemas de injeção/ignição eletrônica convencionais. Aplicam os mesmos sensores e atuadores conhecidos, mas diferenciam-se por permitirem a criação e modificação de todo o mapa de injeção e ignição (CELLA, 2010).

A reprogramação consiste basicamente em alterar os parâmetros relacionados ao combustível, como o tempo de injeção, os parâmetros relacionados a ignição, como o ponto de ignição, assim como outros parâmetros para obter maior desempenho em carros modificados, como, por exemplo, pressão de turbo (ANDREOLI, 2009).

A bancada automotiva foi desenvolvida com a finalidade de ser uma 
plataforma de estudos as aulas de mecânica automotiva, possibilitando uma integração entre a teoria e a pratica durante os ensaios laboratoriais (EDUTEC, 2016).

Neste contexto, o presente trabalho tem como objetivo principal apresentar uma proposta de instalação de um módulo de injeção programável em uma bancada didática de combustão interna de 4 tempos.

\section{MATERIAIS E MÉTODOS}

Foi realizada uma pesquisa técnica referente aos motores de combustão interna e aos módulos de injeção programável disponíveis no mercado, com o objetivo de realizar uma proposta de instalação de um sistema de injeção programável em uma bancada de combustão interna de 4 tempos, para que a mesma possa sofrer alterações no seu sistema de injeção, trazer a visualização das informações de seus sensores e atuadores e estabelecer limites de segurança ao motor.

Também foi realizada uma pesquisa referente aos módulos adicionais que a bancada irá necessitar para a instalação do sistema. São eles: módulo de controle da borboleta eletrônica, módulo Wide band e módulo gerenciador de bobinas.

\section{ESCOLHA DOS MÓDULOS NECESSÁRIOS}

Após um estudo realizado referente aos módulos, optou-se pela escolha do módulo de injeção programável Pandoo Proinject, por possuir uma interface melhor, permitir a verificação de mais dados na tela em um único momento, ter uma maior facilidade em entender as informações da tela, atualização do software do módulo via USB, e por ter um valor mais em conta que o módulo concorrente mesmo necessitando de um módulo adicional para o controle da borboleta eletrônica. Junto ao módulo acompanha um suporte para sua fixação.

Decidiu-se pela escolha do módulo de controle da borboleta eletrônica ETPS Control da Pandoo Performance Parts. Ele possui a função do acelerador eletrônico, o qual é o conversor do pedal do acelerador para a borboleta. O E-TPS Control é responsável por controlar a força do motor de corrente continua que é acoplado no eixo da borboleta para manter a posição desejada de sua abertura. 
Para fazer o condicionamento, medição do teor de oxigênio e a leitura da sonda lambda foi decidido pela escolha do módulo Pandoo Performance Parts Wide band. Este módulo possui uma faixa de funcionamento muito ampla (banda larga), que permite uma resolução com muita precisão.

Para fazer o gerenciamento da faísca de ignição, baseado no sinal gerado pelo módulo de injeção eletrônica programável, optou-se pela escolha do módulo PowerSPARK da Pandoo Performance Parts. Pelo fato do conector da bobina ser de 4 pinos, será utilizado o módulo PowerSPARK $2 \mathrm{CH}$ (dois canais).

\section{INSTALAÇÃO DO SISTEMA}

O módulo de injeção programável Pandoo Proinject possui os mesmos princípios de funcionamento que o módulo original, recebe as informações dos mesmos sensores, e comanda os mesmos atuadores. A diferença na instalação do sistema é que o módulo não possui controle da borboleta eletrônica e gerenciador de bobinas integrado, necessitando de módulos adicionais para seu funcionamento. São eles: módulo de controle da borboleta eletrônica, módulo gerenciador de bobinas. Para uma melhor aferição da mistura ar/combustível é utilizado o módulo Wide band. Na Figura 1 é possível visualizar o esquema da injeção programável Pandoo Proinject. 


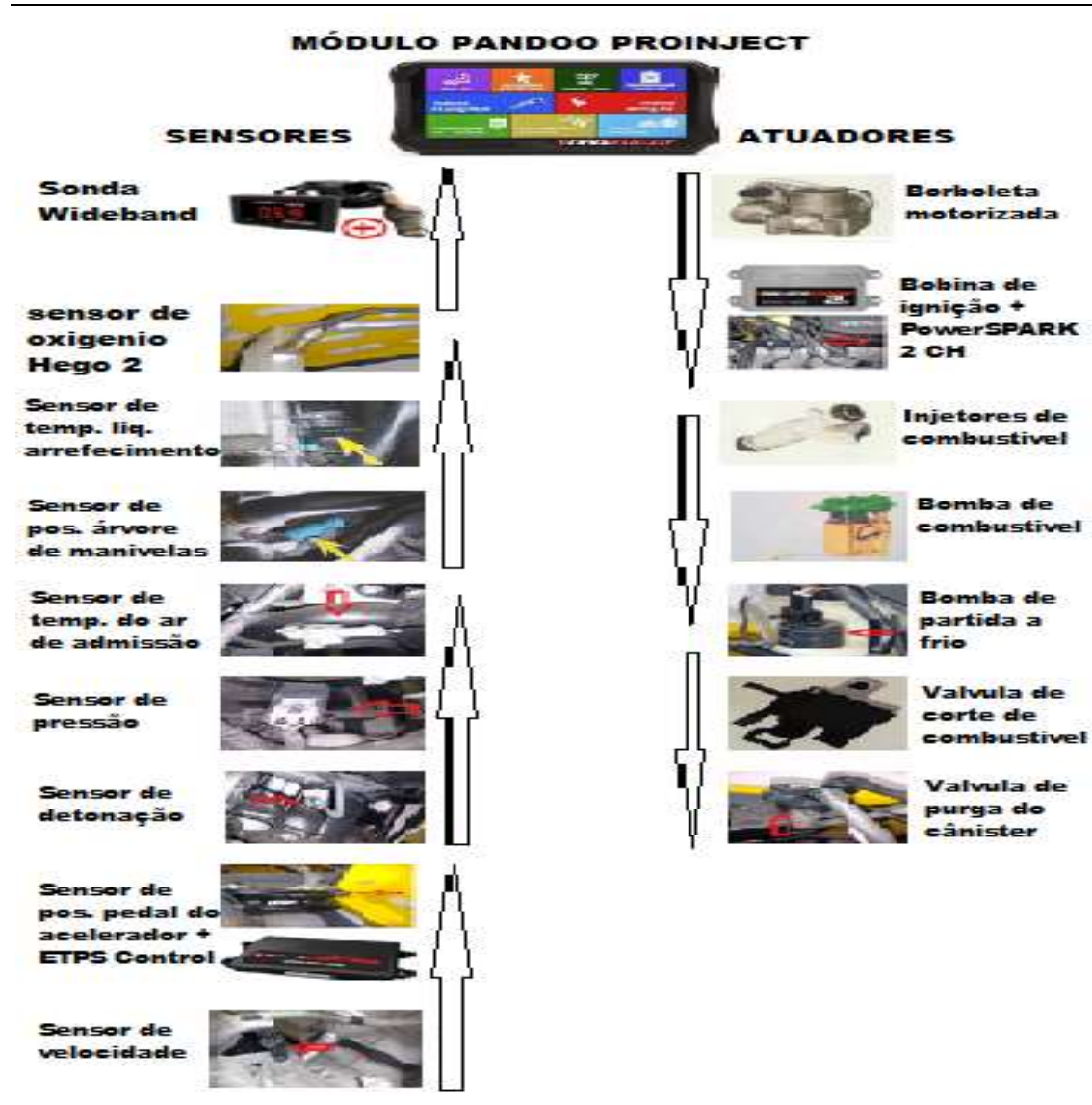

Figura 1. Esquema da injeção programável Pandoo Proinject.

LEVANTAMENTO DE PREÇO DOS MÓDULOS

Foi realizado uma pesquisa no site oficial de vendas dos módulos Pandoo (https://www.lojapandoo.com.br/) na data de 21 de novembro de 2016, referente ao preço dos módulos necessários para a instalação da injeção eletrônica programável. O Quadro 2, lista os módulos, modelos e seus respectivos preços. 


\begin{tabular}{|l|l|l|}
\hline MÓDULO & MODELO & PREÇO (R\$) \\
\hline Injeção Eletrônica Programável & Pandoo Proinject & 2777,00 \\
\hline Controle da Borboleta Eletrônica & Pandoo E-TPS Control & 890,00 \\
\hline Wide band & Pandoo Wide band & 1290,00 \\
\hline Gerenciador de bobinas & Pandoo PowerSPARK 2CH & 440,00 \\
\hline Total & & 5397,00 \\
\hline
\end{tabular}

Figura 2. Levantamento de preço dos módulos.

\section{ANÁLISE DOS RESULTADOS}

A proposta de instalação de uma injeção eletrônica programável em uma bancada de combustão interna foi desenvolvida com o intuito de fazer com que essa bancada didática possa sofrer modificações e melhorias em tempo real no seu funcionamento, assim como uma melhor visualização das informações do funcionamento do motor.

O custo de todos os materiais necessários para a instalação do sistema de injeção eletrônica programável seria de $\mathrm{R} \$$ 5.397,00 (orçamento feito em 21/11/2016 no site oficial de vendas da Pandoo https://www.lojapandoo.com.br/). A instalação deve ser feita por um profissional habilitado na área de mecânica automotiva.

A instalação desta injeção programável se torna viável, pois por se tratar de uma bancada didática que tem por objetivo trazer conhecimento, o sistema fará com que se obtenha mais informações do motor. Através da instalação do sistema, além da bancada possuir o sistema de simulação de defeitos, terá o acompanhamento da qualidade da mistura ar/combustível e fará uma leitura muito mais precisa desta mistura. Além disso, será possível saber a posição do pedal do acelerador, terá a visualização de dados dos sensores de temperatura do motor em ${ }^{\circ} \mathrm{C}$, do sensor MAP em bar e obtenção dos seus valores máximos e mínimos de cada sensor.

Através da instalação deste sistema na bancada, será possível fazer diferentes programações em tempo real no sistema de injeção eletrônica do seu motor. Com essas modificações pode-se ter um melhor aproveitamento de combustível, melhoramento no torque e potência, maior giro final do motor, maior 
segurança na parte mecânica, limitadores de pressão e rotação (corte de ignição), limitador de temperatura (limitador de rotação especial), alimentação de combustível ajustável para qualquer faixa de trabalho, entre outros.

Através do Pandoo ECU Manager será possível fazer a configuração e visualização de mapas como por exemplo de rotação, sonda lambda, sensor MAP, TPS, temperatura do motor e temperatura do ar. Poderá ser feita a correção por MAP e por sonda lambda, configurar a injeção, proteções do motor, criar novos mapas, entre outros.

\begin{tabular}{|c|c|c|}
\hline FUNÇÕES & $\begin{array}{l}\text { BANCADA ANTES DA } \\
\text { INSTALAÇÃO DOS } \\
\text { MÓDULOS }\end{array}$ & $\begin{array}{l}\text { BANCADA APÓS A } \\
\text { INSTALAÇÃO DOS } \\
\text { MÓDULOS }\end{array}$ \\
\hline Simulador de defeito dos sensores. & SIM & SIM \\
\hline $\begin{array}{l}\text { Visualização da leitura da mistura } \\
\text { ar/combustível. }\end{array}$ & NÃO & SIM \\
\hline $\begin{array}{l}\text { Leitura da mistura ar/combustível em } \\
\text { banda larga. }\end{array}$ & NÃO & SIM \\
\hline $\begin{array}{l}\text { Informação do sensor MAP (bar) através } \\
\text { de uma tela de visualização. }\end{array}$ & NÃO & SIM \\
\hline $\begin{array}{l}\text { Visualização da informação de } \\
\text { temperatura do motor em }{ }^{\circ} \mathrm{C} \text {. }\end{array}$ & NÃO & SIM \\
\hline $\begin{array}{l}\text { Visualização da informação da } \\
\text { temperatura do ar de admissão em }{ }^{\circ} \mathrm{C} .\end{array}$ & NÃO & SIM \\
\hline $\begin{array}{l}\text { Visualização da informação da } \\
\text { temperatura do óleo em }{ }^{\circ} \mathrm{C} \text {. }\end{array}$ & NÃO & SIM \\
\hline $\begin{array}{l}\text { Visualização da informação da } \\
\text { temperatura da água em }{ }^{\circ} \mathrm{C} .\end{array}$ & NÃO & SIM \\
\hline $\begin{array}{l}\text { Proteção para rotação, temperatura, } \\
\text { pressão do óleo e combustível. }\end{array}$ & NÃO & SIM \\
\hline Configuração do mapa de injeção. & NÃO & SIM \\
\hline Correções no avanço de ignição. & NÃO & SIM \\
\hline Acesso aos mapas de injeção e ignição. & NÃO & SIM \\
\hline $\begin{array}{l}\text { Visualização de mapas de informação de } \\
\text { sensores e atuadores. }\end{array}$ & NÃO & SIM \\
\hline $\begin{array}{l}\text { Configuração de até } 5 \text { mapas de injeção } \\
\text { diferentes. }\end{array}$ & NÃO & SIM \\
\hline
\end{tabular}

Figura 3. Funções da bancada.

A fixação do módulo de injeção eletrônica precisa ficar em um local de fácil 
visualização, pois é através dele que é feita toda a programação e a obtenção das informações do motor. Após a instalação e fixação de todos os componentes, a bancada de combustão interna ficará de acordo com a Figura 4.

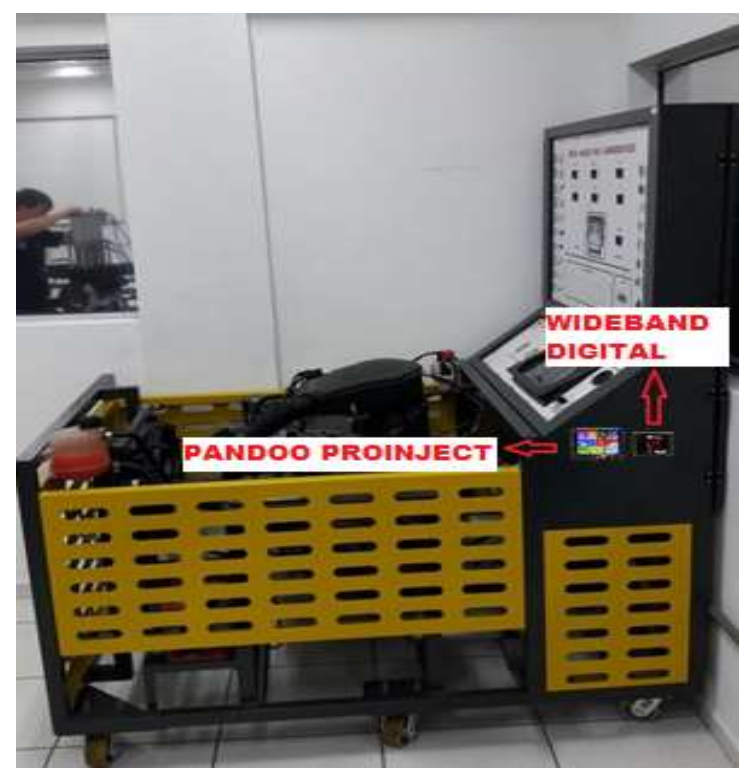

Figura 4. Componentes instalados.

\section{CONCLUSÕES}

Foi feita a escolha do módulo de injeção programável, módulos auxiliares e a proposta de instalação em uma bancada didática de combustão interna de 4 tempos. Através desta instalação é possível obter a visualização das informações dos sensores do motor e o controle dos seus atuadores, estabelecer limites de segurança, realizar diferentes tipos de configurações de trabalho, ter acesso aos mapas de injeção e ignição e poder analisá-los e configura-los.

Fica como perspectivas futuras a instalação do sistema proposto na pesquisa, para que se possa pôr em prática seu funcionamento, realizar diferentes programações no sistema de injeção do motor, obter suas informações em funcionamento e analisá-las. 


\section{REFERÊNCIAS}

ANDREOLI, Alexandre Giordani. Injeção Eletrônica Programável para Automoveis: 2009. 129 f. Projeto de Diplomação (Graduação em Engenharia Elétrica) Universidade Federal do Rio Grande do Sul, Porto Alegre.

BOSCH, Robert. Manual de Tecnologia Automotiva. 25 Ed. São Paulo: Edgard Blucher, 2005.

BRUNETTI, Franco. Motores de Combustão Interna. 1 Ed. São Paulo: Edgard Blucher, 2012.

FAGGI, Rodrigo. Formação de mistura ar combustível em motores de ignição por faísca a quatro tempos. 2012. 60 f. Monografia (Especialização) - Instituto Mauá de Tecnologia, São Caetano do Sul.

CELLA, Igor Eriberto et al. Regulagem da unidade de comando eletrônico programável modelo fuel-tech para carros de competição. In: CONGRESSO NACIONAL DE ENGENHARIA MECANICA, 6, 2010. Campina Grande. Anais... São Luiz: Instituto Federal de Educação, Ciência e Tecnologia do Maranhão, 2010. p. 1 - 9.

EDUTEC. Manual do sistema elétrico de falhas: Bancada de combustão interna de 4 tempos. Curitiba. [20--]. 23 p. 